\title{
A comparison of the length and width of static inked two-dimensional bare footprints found on a hard compared to a soft surface.
}

\section{Michael Curran}

The University of Northampton, University Drive Northampton, UK. NN2 5PH.

\section{Mike.Curran@Northampton.ac.uk}

Isabelle Holmes

The University of Northampton, University Drive Northampton, UK. NN2 5PH. isabelleholmes@,aol.com

\section{Corresponding author}

\section{Michael Curran}

The University of Northampton, University Drive Northampton, UK. NN2 5PH.

\section{Mike.Curran@Northampton.ac.uk}

\begin{abstract}
In forensic intelligence-gathering it would be useful to evaluate if there are differences between static inked bare footprints captured on hard surfaces compared to soft surfaces. This was undertaken using samples from 30 undergraduate students. Initially a static footprint was taken for each participant on a hard surface and this was followed by a static footprint on a soft surface. On both occasions, the participants stood on an inkless mat and then on reactive paper, creating a twodimensional print. The Reel method was used to analyse each footprint and the print was measured to see whether a difference existed between length and width (forefoot and rearfoot width) on a hard surface compared to a soft surface. The conclusion from this study was there is a statistically significant increase in length and width of a static bare footprint on a soft surface as opposed to a hard surface. If a forensic footprint examiner compares static bare footprints found on a soft surface and compares them to a static bare footprint of the same foot taken later, then the increase in both length and width of the footprints on a soft surface should be considered in the evaluation.
\end{abstract}

Keywords

Bare; footprints; soft; hard; surface; static;

Highlights

An increase in the mean length of a bare footprint on a soft surface. 
An increase in the mean width (forefoot and heel) of a bare footprint on a soft surface.

Statistically significant increase in length of a bare footprint on a soft surface.

Statistically significant increase in width (forefoot and heel) of a bare footprint on a soft surface.

\section{Formatting of funding sources}

This research did not receive any specific grant from funding agencies in the public, commercial, or not-for-profit sectors.

Novelty statement.

In forensic casework involving analysis of static bare static footprints there is no literature about potential differences in length and width of static bare footprints found on soft and hard surfaces. This study demonstrates that there is a statistically significant increase in both length and width of a two-dimensional static inked bare footprint when measured on a soft surface as opposed to a hard surface.

\section{1) Introduction}

There has been increasing interest in the potential of bare footprints being an aid to identification [1]. Studies have demonstrated that bare footprints are highly individual because of differences in the foot dimensions of the people who created them.

According to Di Maggio and Vernon [2] and Reel et al. [3] bare footprints can be static or dynamic where static prints are associated with standing and dynamic prints with walking or running. Vernon et al. report that experience has shown that the dynamic form of bare footprints typically presents with two features not usually seen in static prints; namely inner dark and outer ghosting areas at the posterior (heel) and various anterior (toe) areas. A footprint successfully recovered from a scene of crime is known as the 'questioned' print and an inked footprint taken from an individual linked with the incident is referred to as the 'known print' and has been used in the literature as a comparison with the 'questioned print' [1]

Bodziak [4] found that there are three types of footprints that can be found at the scene of a crime: (1) the impression left in an insole of a shoe, (2) a true bare footprint, and (3) a foot that had a sock on. Bare footprint comparisons have been widely accepted as a method that can assist with the process of identification. According to Barker and Scheuer [5], in the western world, there is a role for the bare footprint in forensic investigations. Kennedy et al. [6] considered the uniqueness of bare footprints as an aid to identification in a study which, at the time of publication, had utilized 24,000 footprints collected from 12,000 volunteers under standardised conditions. Although he concluded that footprints were individual, Di Maggio and Vernon (2) argue that these standardised conditions do not reflect real-world 
situations such as interpopulation differences and it may be that the prints are individual in form but may not be unique. Krishan et al. [7] considered the individuality of footprints in a Gujjar population in North India, particularly considering shape, alignment, creases, size, cuts, cracks, and pits to determine whether these characteristics were individual. The study involved 1040 adult males between 18 and 30 years of age. Bilateral prints $n=2080$ were taken. Each print was taken using ink that was applied to the plantar surface of the foot after which the participant would step onto white plain paper and then repeat with the opposite foot. The footprints were shown to be highly individual and showed a link with personal identity. Moorthy and Sulaiman [8] conducted a study involving 200 males and 200 females Malaysian participants between 18 and 60 years of age. Eight hundred bilateral prints were collected in total. The results of this study concluded that each footprint had individual characteristics, therefore supporting the results found by both Krishan et al. [7] and Kennedy et al. [6]

Mathieson et al [9] investigated the connection between a foot type and foot pathology by collecting footprint parameters from static and dynamic footprints. The study concluded that in dynamic prints the parameters altered significantly, compared to those in the static print. This was also supported by Curran et al [10] who considered the difference between the base of gait and the angle of gait in both a dynamic and static footprint. They found that there was no significant difference in the dynamic and static footprint comparisons in the angle of gait, however there was a significant difference in the dynamic and static footprint comparison in the base of gait. It was also suggested that the static footprints revealed prediction of a dynamic function when assessing the angle of gait and the base of gait in normal subjects.

DiMaggio and Vernon [2] stated that there are two forms of surface at a crime scene these include two dimensional and three-dimensional surfaces. The threedimensional footprints are made in a softer substrate, for example sand or dirt. However, the differences between parameters of these prints have not been investigated.

This study aimed to investigate whether there are differences between static inked bare footprints captured on hard surfaces compared to soft surfaces This information could be used as an aid for gathering intelligence about whether a person may be associated or disassociated from a crime scene.

2) Materials and method

An opportunistic sample of 30 participants was recruited from the student population of a University in the UK. Ethical approval for the study was obtained from the Faculty Ethics Committee at the study University. Participants were fully informed of the study by one of the authors and each participant gave their consent to participate in the study. The inclusion and exclusion criteria were that participants were aged over 18 and had no history of foot or leg deformity or surgery to the limb.

2.1) Method used for obtaining a standing static footprint. 
Following the protocol described by Reel et al. [1] an inkless pad was placed on the floor. Participants placed their foot onto the inkless pad in their natural stance position and held it for three seconds. They then transferred their foot onto the treated paper next to the inkless mat in their natural stance, thus creating a twodimensional static bare footprint. In addition to the Reel protocol the foot was wiped beforehand with antiseptic wipes to prevent cross infection, though Reel et.al [1] reported there have been no known allergies or cross infection associated with this. Initially this process was done for footprints on a hard surface and then repeated on a soft surface which was carpet. This is represented in fig 1. and fig 2. The manufacturers specification for the carpet states that it is made from $100 \%$ polypropylene, with a pile height of $12.5 \mathrm{~mm}$ and a total height of $15.5 \mathrm{~mm}$ and described as soft feel. The actual sample of carpet is represented in fig 3 . 


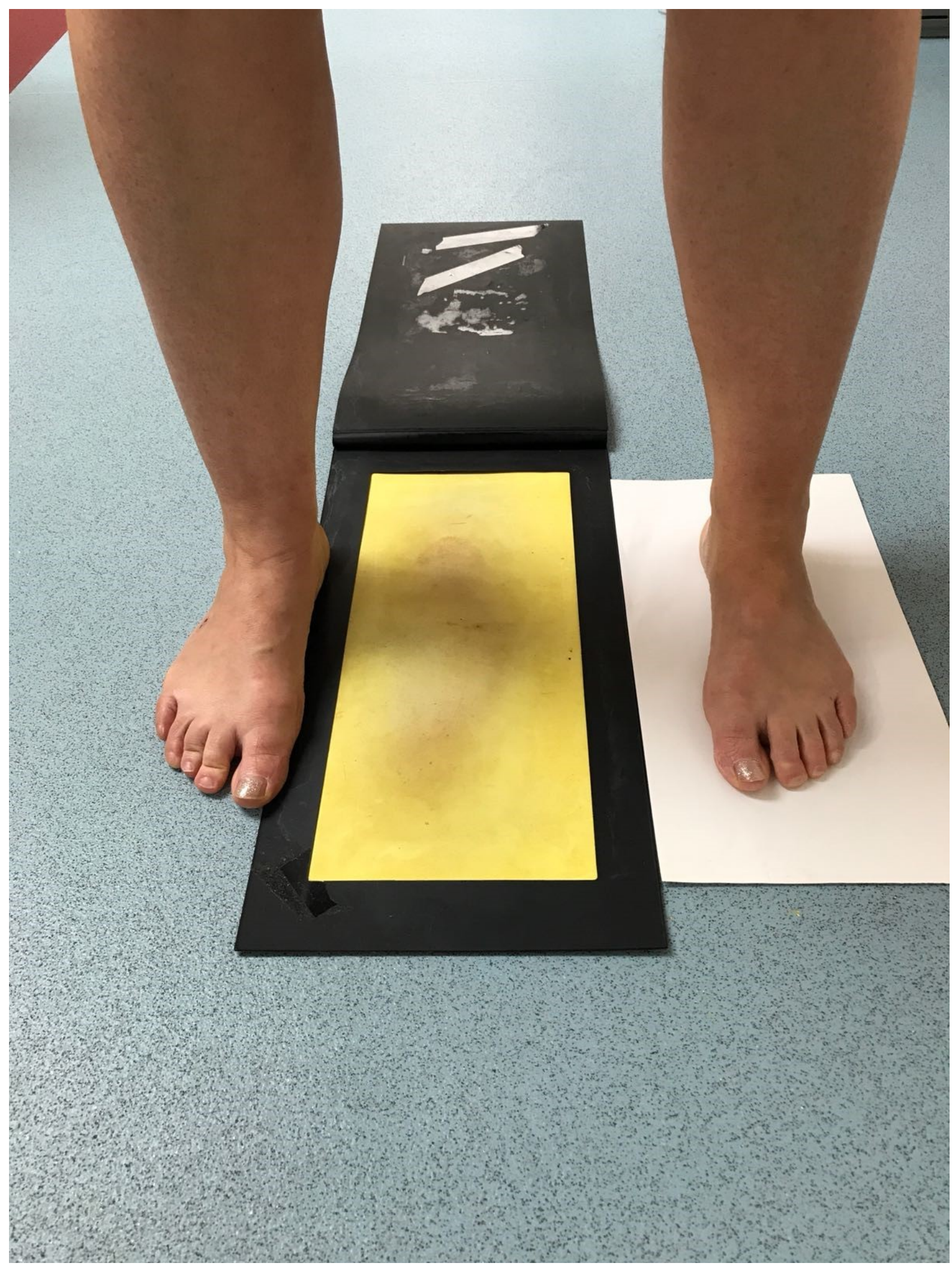

Fig1 Process of obtaining a standing bare footprint on a hard surface 


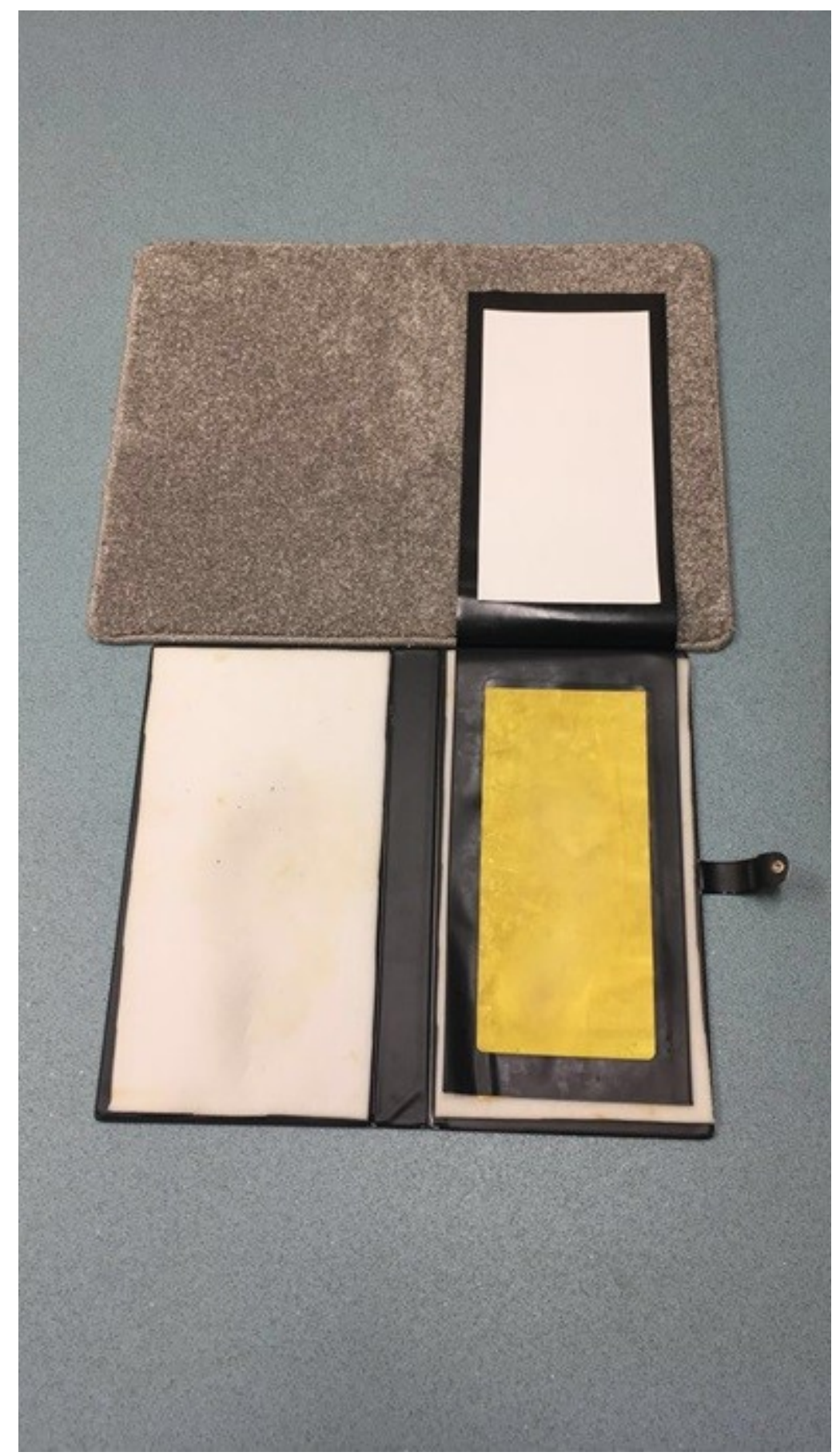

Fig 2 Process of obtaining a standing bare footprint on a soft surface (carpet) 


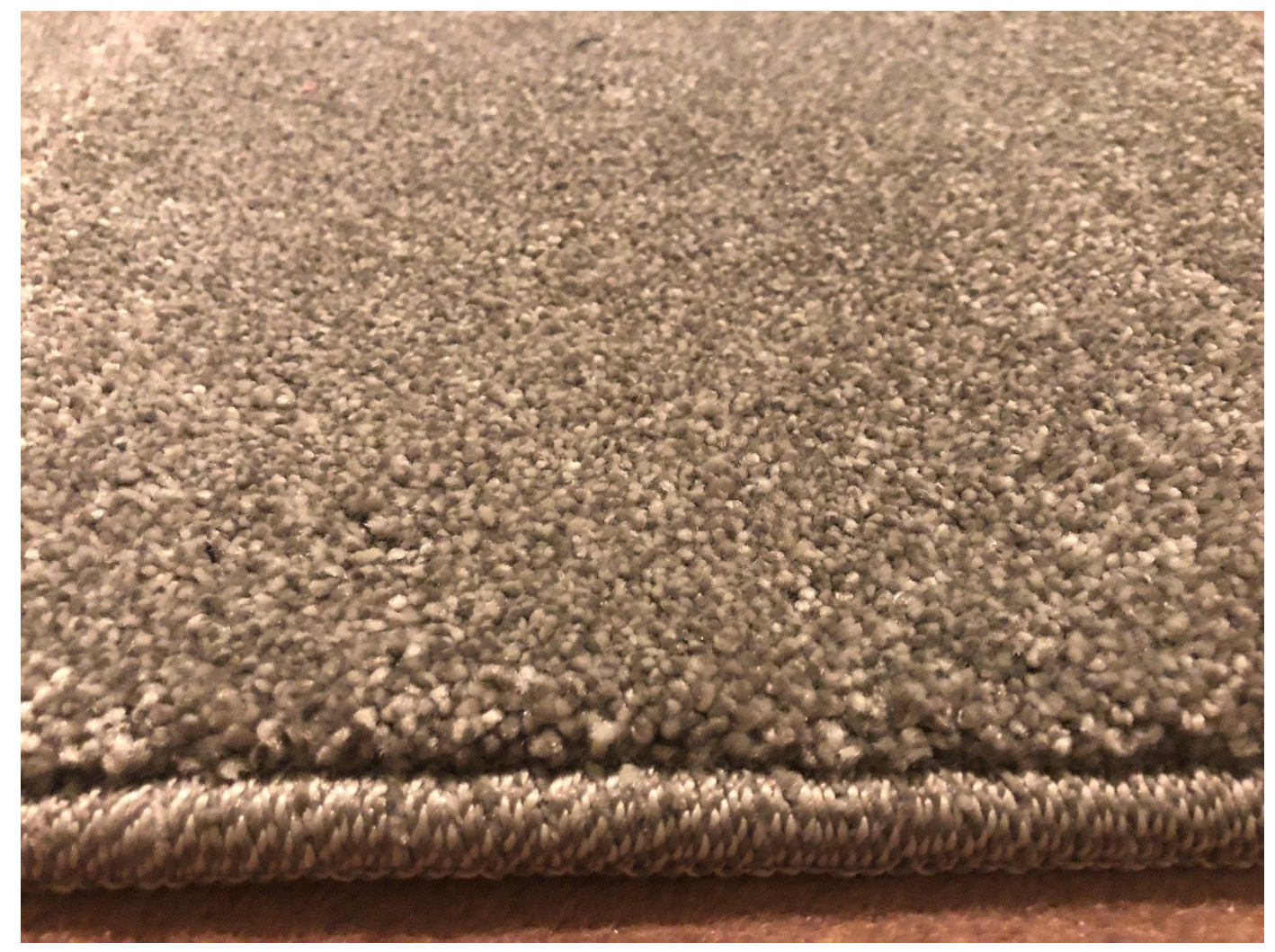

Figure 3 Carpet used in the study

\subsection{Footprint Analysis: Scanning GIMP and Reel Method}

Following the guidelines set out by Reel [1], Each static bare footprint was scanned at 150 dots per inch and saved as a JPEG file. Each footprint was analysed using GNU Image Manipulation Program(GIMP) 2.8 software.

As shown in fig 3 , the central axis of the footprint was found and then the print was rotated until the central axis was vertical. A line was then drawn skimming the lowest heel pixel and crossing the central axis. The highest pixel of the most distal aspect of the foot, regardless to whether it represented the first or second toe as was marked. A line was then drawn from the central aspect of the calcaneus to the most distal part of the foot that had been marked and thus giving a measurement of longest possible footprint length. A measurement was then taken for foot MPJ width at the forefoot and calcaneum width at the rearfoot as shown in fig 3. These measurements were recorded on a Microsoft Excel spreadsheet. 


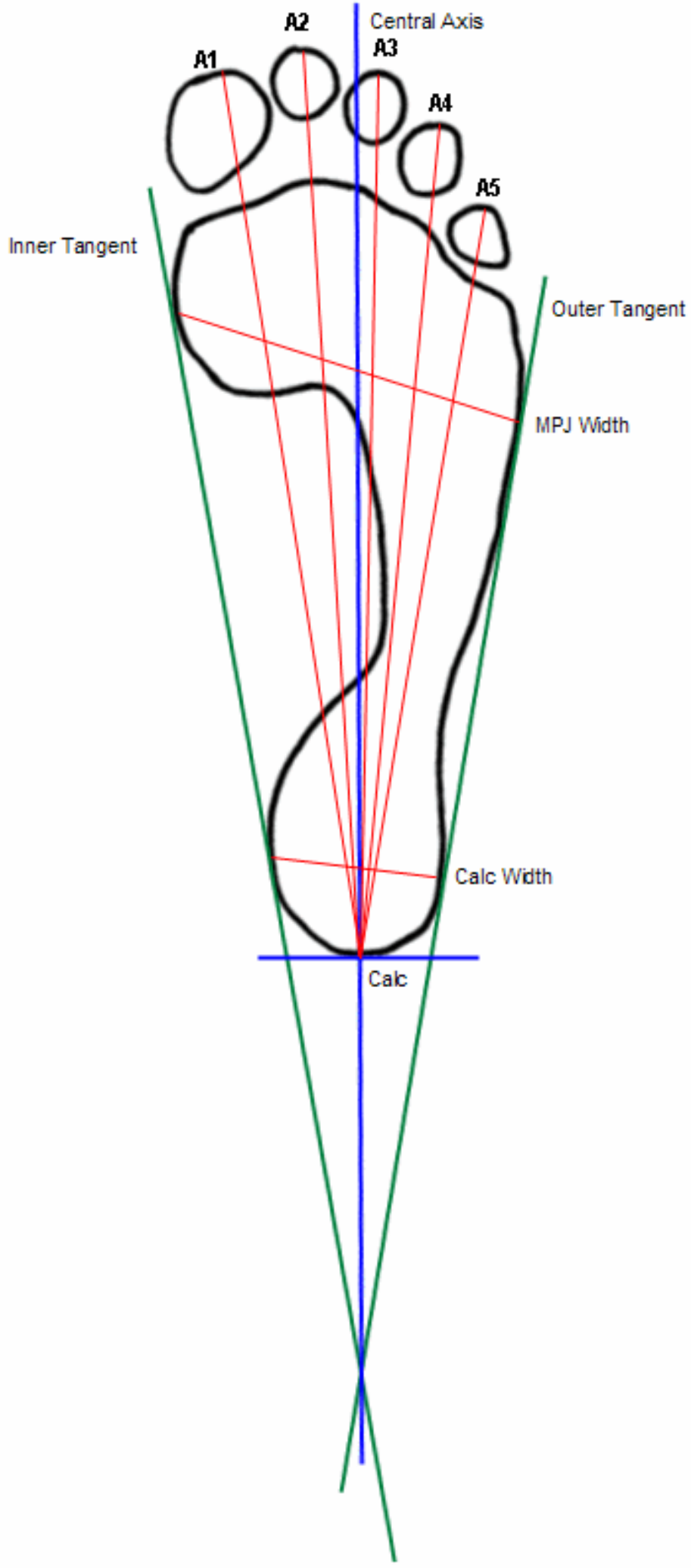

Fig 3.

Reel Method 
3) Results

\subsection{Sample Characteristics.}

There were 30 participants in the study comprising eight males and twentytwo females aged between eighteen and fifty.

3.2 Analysis of the results using descriptive statistics

\begin{tabular}{|l|l|l|l|}
\hline & Mean & $\mathbf{N}$ & Std. deviation \\
\hline $\begin{array}{l}\text { Length on } \\
\text { hard surface } \\
\text { (mm) }\end{array}$ & 233.980 & 30 & 14.2803 \\
\hline $\begin{array}{l}\text { Length on } \\
\text { Soft surface } \\
(\mathrm{mm})\end{array}$ & 239.803 & 30 & 14.4737 \\
\hline
\end{tabular}

Table 1

Summary table for length of a footprint.

\begin{tabular}{|l|l|l|l|}
\hline & Mean & N & Std. deviation \\
\hline $\begin{array}{l}\text { Width at the } \\
\text { forefoot on a } \\
\text { hard surface } \\
\text { (mm) }\end{array}$ & 87.643 & 30 & 6.4782 \\
\hline $\begin{array}{l}\text { Width at the } \\
\text { forefoot on a } \\
\text { soft surface } \\
\text { (mm) }\end{array}$ & 93.320 & 30 & 5.7194 \\
\hline
\end{tabular}

Table 2

Summary table for width of a footprint measured at forefoot.

\begin{tabular}{|l|l|l|l|}
\hline & Mean & N & Std. deviation \\
\hline $\begin{array}{l}\text { Width at the } \\
\text { rearfoot on a } \\
\text { hard surface } \\
\text { (mm) }\end{array}$ & 48.230 & 30 & 4.4426 \\
\hline $\begin{array}{l}\text { Width at the } \\
\text { rearfoot on a } \\
\text { soft surface } \\
(\mathrm{mm})\end{array}$ & 54.530 & 30 & 4.7611 \\
\hline
\end{tabular}


Table 3

Summary table for width of a footprint measured at the rearfoot.

Table 1 shows that when comparing length of the footprint on a hard compared to a soft surface, the mean footprint length on a hard surface was 233.980 (SD 6.4782) and the mean footprint length on a soft surface was 239.803 (SD 14.4737). indicating an increase in footprint length on a soft surface compared to a hard surface.

Table 2 shows that when comparing width of the footprint at the forefoot on a hard compared to a soft surface, the mean footprint width on a hard surface was 87.643 (SD 6.4782) and the mean footprint width on a soft surface was 239.803 (SD 5.7194). indicating an increase in footprint width on a soft surface compared to a hard surface.

Table 3 shows that when comparing width of the footprint at the rearfoot on a hard compared to a soft surface, the mean footprint width on a hard surface was 48.230 (4.4426) and the mean footprint width on a soft surface was 54.530 (SD 4.7611). indicating an increase in footprint width on a soft surface compared to a hard surface.

3.3 Analysis of the results using a paired test.

The results of a paired t test showed that there was a highly statistical significant difference in the length of a static bare footprint on a soft surface compared to a hard surface $(p=.000)$

A paired $t$ test was then repeated for width of a static bare footprint when measured at the forefoot and then at the rearfoot. Both these $t$ tests showed a highly statistical significant increase in width of the footprint taken on a soft surface compared to a hard surface. $(p=.000)$.

\section{4) Discussion}

When a static bare footprint has been recovered from a crime scene, a forensic footprint examiner could be asked to investigate whether that bare footprint could be associated with a perpetrator of a crime. The findings from the study show that both the length and width at the forefoot and rearfoot of static bare footprints increase on a soft surface compared to a hard surface and that these differences are statistically significant. There are no previous studies that focus on the same area as this study therefore comparing the findings of this study with previous work is not possible. Di Maggio and Vernon [2] state that soft tissue expansion of the foot occurs on weight bearing and this is likely to be dependent to some degree on the substrate surface. While it may be suggested that the findings from this study are obvious that testing and subsequent publication of this finding is not required it can be the case that prior beliefs can be disproved through research which can be known to demonstrate unexpected results.

A limitation of the study could be that the bare footprints were taken with the inkless paper placed on top of the carpet. Usually the bare footprint would come 
directly in contact with the carpet to form the footprint. However, when the inkless paper was placed underneath the carpet then the footprint was found to be of poor quality and not useable. This study contains findings from one type of soft surface a piece of carpet made from $100 \%$ polypropylene, with a pile height of $12.5 \mathrm{~mm}$ and a total height of $15.5 \mathrm{~mm}$ and it would be interesting to vary the type of soft surface to see if and to what extent the findings differ. This study used static bare footprints, it would be useful to investigate dynamic bare footprints to see if and to what extent the findings differ.

The result was derived from a single sample of 30 participants. A larger sample would result in narrower prediction intervals and therefore improve the precision of the findings. Validation studies are needed to confirm these findings using other samples from the same population and from populations with differing characteristics to ensure its generalisability.

\section{5) Conclusion}

This study focused on one type of soft surface and one kind of carpet and measured the width of the foot at the forefoot and the rearfoot. However, the findings from this study demonstrated that the length and width of a static bare footprint does change, and that this change is statistically significant. The results showed that both the length and width (forefoot and rearfoot) of a static bare footprint increases significantly when the foot has been placed on to a soft surface a piece of carpet manufactured from $100 \%$ polypropylene, with a pile height of $12.5 \mathrm{~mm}$ and a total height of $15.5 \mathrm{~mm}$ as opposed to a hard surface. If a forensic footprint examiner compares static barefoot prints found on a soft surface and compares them to a static bare foot print of the same foot taken later, then the increase in both length and width of the footprints should be considered in the evaluation.

\section{References}

1.Vernon, W. A Work Based Observational Action Research Project Involving Males of Working Age to Determine the Cause of the Inner Darker Areas and Outer Lighter Areas of Ghosting Seen in Two-dimensional Dynamic Bare Footprints. MSc Dissertation, University of Huddersfield, 2015.

2. DiMaggio, J.; Vernon, W. Forensic Podiatry: Principles and Methods; CRC Press: Boca Raton, FL, 2017.

3.Reel S, Rouse, S, Vernon, W, Dohery, P Estimation of stature from static and dynamic footprints. Forensic Sci Int,219, (283) (2012).

4. Bodziak, W. J. Footwear Impression Evidence: Detection, Recovery, and Examination, 2nd ed. CRC Press: Boca Raton, FL, 1999.

5.Barker, S. L.; Scheuer, J. L. Predictive Value of Human Footprints in a Forensic Context. Med. Sci. Law 1998, 38 (4), 341-346. 
6. Kennedy, R. B., Chen, S., Pressman, I. S., Yamashita, A. B. \& Pressman, A. E. A large-scale statistical analysis of barefoot impressions. J For. Sci. (2005) 1071-1080.

7. Krishan, K. Individualizing Characteristics of Footprints in Gujjar's of North IndiaForensic Aspects. For. Sci. Int. 2007, 169 (2-3), 137-144.

8. Moorthy, T. N.; Sulaiman, S. F. B. Indivualizing Characteristics of Footprints in Malaysian Malays for Person Identification from a Forensic Perspective. Egyptian J. For. Sci.5 (1) (2015) 13-22

9.Mathieson, I., Upton, D. and Birchenough, A. (1999). Comparison of footprint parameters calculated from static and dynamic footprints. The Foot, 9(3), pp.145149.

10. Curran, S., Upton, D. and Learmonth, I. (2005). Dynamic and static footprints: comparative calculations for angle and base of gait. The Foot, 15(1), pp.40-46. 